\title{
¿Qué se dice de la mujer y el hombre en el español de Chile?: estudio exploratorio de las combinaciones frecuentes de los vocablos mujer y hombre en un corpus de referencia estratificado
}

\author{
María Natalia Castillo Fadić* \\ Pontificia Universidad Católica de Chile, Chile
}

\begin{abstract}
Resumen
El vocablo mujer presenta alta frecuencia $\left(\mathrm{F}_{\mathrm{i}}\right)$, dispersión (D) y uso (U) en el Corpus Básico del Español de Chile $1\left(\mathrm{~F}_{\mathrm{i}}=340, \mathrm{D}=0,73\right.$ y $\mathrm{U}=248,2)$. Si se lo compara con el vocablo hombre, éste aparece con índices mayores $\left(\mathrm{F}_{\mathrm{i}}=502, \mathrm{D}=0,8 \mathrm{y} \mathrm{U}=401,6\right)$, lo que podría atribuirse a su posibilidad de representar tanto a los individuos humanos masculinos como a los humanos en general; esta hipótesis parece confirmarse por el hecho de que la distribución de ambos vocablos por mundo es similar en cuatro de cinco mundos (Drama, Narrativa, Técnico-Científico y Prensa), pero disímil en Ensayo; más aún, el $9 \%$ de las ocurrencias de hombre y de mujer tiene lugar en Drama y el $14 \%$ de las ocurrencias de cada vocablo se presenta en TécnicoCientífico, con una distribución idéntica; en Ensayo, en cambio, tiene
\end{abstract}

Para correspondencia, dirigirse a: María Natalia Castillo Fadić (mcastilf@uc.cl). Facultad de Letras, Pontificia Universidad Católica de Chile, Campus San Joaquín. Av. Vicuña Mackenna 4860, Macul. Santiago, Chile. Teléfono: (+56) 223547859.

1 María Natalia Castillo Fadić, 2012 (C). 
lugar un $26 \%$ de las ocurrencias de hombre, el doble de las de mujer en el mismo mundo, justamente por tratarse del género donde con mayor prominencia hombre se emplea con un significado que abarca tanto a hombres como a mujeres de la especie humana.

La diferencia en los índices estadísticos de uno y otro, por tanto, no puede interpretarse directamente como una manifestación de la disímil representación de la mujer y el hombre en el español de Chile. No obstante, las unidades léxicas con las que cada uno de estos vocablos se combina, especialmente cuando se trata de unidades predicativas, sí pueden dar indicios respecto de cómo concibe la comunidad a hombres y a mujeres.

Palabras clave: estadística léxica, léxico-sintaxis, lingüística de corpus, español de Chile, estudios de género.

\section{WHAT DO GENRES SAY ABOUT WOMEN AND MEN IN CHILEAN}

SPANISH? AN EXPLORATORY STUDY OF THE FREQUENT COMBINATIONS OF THE WORDS WOMAN AND MAN IN A STRATIFIED REFERENCE CORPUS

Abstract

The term mujer occurs with a high frequency (if), dispersion (D) and use (U) in the Basic Corpus of Chilean Spanish $\left(\mathrm{F}_{\mathrm{i}}=340, \mathrm{D}=\right.$ $0,73$ and $U=248,2)$. The word man on the other hand, presents a higher rate of occurrence $(\mathrm{Fi}=502, \mathrm{D}=0.8$ and $\mathrm{U}=401.6)$, which is likely to be since both males and females are referred to as men when denoted as humans beings. This hypothesis seems to be confirmed by a similar distribution in four out of five textual genres (Drama, Narrative, Technical-Scientific and Press), but quite a disparate one in the Essay. 9\% of the man and woman occurrences are recorded in Drama and $14 \%$ of the occurrences of each term appear in Technical-Scientific texts, with an identical distribution. Unlike these, the occurrences of man reaches $26 \%$ in the Essay, twice the rate of woman, precisely because in this genre the term man is more frequently used to encompass both as members of the human species. These statistic differences cannot be directly interpreted as an uneven representation of men and women in Chilean Spanish. However, the combinations these lexical units establish, especially when it comes to predictable units, may give some hints to the conception of men and women in society.

Keywords: Lexical statistics, lexico-syntaxis, corpus linguistics, Chilean Spanish, gender studies.

Recibido: 10/11/18 Aceptado: 11/01/19 


\section{INTRODUCCIÓN}

En el marco del actual interés público por la inclusión social de la mujer a través del lenguaje ( $c f$. , por ejemplo, Marín 2018 y Manrique Sabogal 2012) y en medio de la discusión acerca de si la lengua española la invisibiliza o no al usar el masculino como género no marcado, nos hemos propuesto hacer una contribución al tema desde la lingüística de corpus.

Siendo la lengua espejo de la cosmovisión de los pueblos, un cambio lingüístico requiere de un cambio cultural que se produzca y se asiente en el tiempo; y como nos muestran también los datos empíricos históricos y la observación de procesos homólogos contemporáneos, hay niveles más permeables al cambio y otros menos permeables: el morfológico, que se busca modificar, negando la posibilidad de usar el masculino como género no marcado, ha sido históricamente y en diferentes lenguas el menos permeable, junto con el fonológico; el léxico, en cambio, no solo es el más permeable, sino también el más cercano a la cosmovisión de los hablantes; los usos de ese léxico, sus combinatorias, las predicaciones, no solo entregan luces sobre la mirada de un pueblo acerca de una realidad, sino que también pueden ser el punto de más fácil intervención cuando se desea erradicar una asociación conceptual indeseada o políticamente incorrecta.

Ahora bien, una intervención en este nivel supone conocer previamente el estado de las cosas. Es por ello que nos preguntamos en este artículo qué se dice del hombre y la mujer o, en términos lexicosintácticos, qué se predica de ambos cuando funcionan como sustantivos. Este estudio lexicoestadístico y lexicosintáctico, por tanto, se centra en las combinaciones frecuentes de los vocablos mujer y hombre en un corpus de referencia estratificado: el Corpus Básico del Español de Chile (Castillo Fadić 2012). El foco está en la combinación de cada uno con los modificadores nominales ubicados a la derecha de cada uno de estos sustantivos: adjetivos, complementos y locuciones.

Se trata de un trabajo descriptivo y predominantemente cuantitativo, exploratorio, centrado en los niveles léxico y sintáctico, sincrónico y sintópico, de naturaleza empírica, finalidad pura e implicancias aplicadas, que utiliza herramientas de lingüística de corpus y lingüística computacional y que considera el factor social. Su objetivo general es precisar qué modificadores nominales determinan a los vocablos mujer y hombre en el español de Chile. Los específicos son: a) describir estadísticamente la distribución de mujer y hombre por mundo; b) describir estadísticamente las combinaciones léxicas frecuentes de cada vocablo; c) abstraer las construcciones sintácticas más frecuentes que modifican a los vocablos mujer y hombre, a partir del 
análisis por clusters; d) precisar qué modificadores nominales determinan al vocablo mujer con $\mathrm{F}_{\mathrm{i}} \geq 2$; e) precisar qué modificadores nominales determinan al vocablo hombre con $\mathrm{F}_{\mathrm{i}} \geq 2$; f) comparar y contrastar los modificadores nominales frecuentes de ambos vocablos; g) analizar las combinaciones lexicosintácticas frecuentes para verificar la existencia de unidades fraseológicas, colocaciones o restricciones léxicas que incluyan o modifiquen a uno de estos vocablos o a ambos; h) contribuir al estudio del español de Chile; i) sentar bases para contribuir a los estudios de género desde la lingüística.

Dado el alto uso de los vocablos hombre y mujer en el español de Chile ( $c f$. Castillo Fadić, en referato), ambos deberían presentar escasas restricciones combinatorias y mínima dependencia de variables externas. Se espera, por tanto, $\mathrm{y}$ a modo de hipótesis de base, un alto número de modificadores nominales con $\mathrm{F}_{\mathrm{i}}=1$ y un bajo número de modificadores nominales con $\mathrm{F}_{\mathrm{i}}>1$. Como segunda hipótesis, se espera obtener más predicaciones con valor semántico-pragmático positivo para hombre que para mujer $\mathrm{y}$, a la inversa, como tercera hipótesis, se espera obtener más predicaciones con valor semántico-pragmático negativo para mujer que para hombre.

La investigación pretende ayudar a identificar la imagen de la mujer y el hombre en la sociedad chilena contemporánea, a partir de sus representaciones lingüísticas. Esta identificación puede ser el punto de partida para abordar la posibilidad de introducir mejoras en las políticas de inclusión, equidad e igualdad de género. Desde las políticas lingüísticas, esta mejora podría considerar las estructuras adjetivas en las políticas de género y no solo las centradas en flexión de género gramatical.

\section{FUNDAMENTO TEÓRICO}

\subsection{LEXICOESTADÍSTICA}

La lexicoestadística o estadística léxica estudia cuantitativamente el nivel léxico. Puesto que opera a nivel de lengua, no se detiene en las unidades de texto o palabras, sino que se enfoca en las unidades de léxico o vocablos. Mientras las palabras -variantes-corresponden a las realizaciones a nivel textual, los vocablos -invariantes- representan un paradigma de palabras que comparten base léxica y contenido semántico. En el caso de las formas nominales, el vocablo suele corresponder a la unidad léxica en masculino, 
en singular y en su forma primitiva o no derivada; las palabras, en cambio, se forman a partir de la flexión o derivación de una base léxica común. En lo concerniente a las unidades léxicas en estudio, en la medida en que mujer y hombre no presentan flexión de género -puesto que la noción de femenino o masculino está incluida en la base respectiva-, las palabras por medio de las cuales se realizan ambos vocablos solo pueden experimentar flexión de número o derivaciones. Para hacer factible el conteo estadístico, metodológicamente se emplea también el concepto de tipo, unidad léxica intermedia que funciona como etiqueta para todas las ocurrencias de palabras que, agrupadas bajo un mismo vocablo por sus características morfológicas y semánticas, comparten un mismo significante.

La estadística léxica supone la medición de índices estadísticos. El más simple es el de la frecuencia: esta puede ser absoluta $\left(\mathrm{F}_{\mathrm{i}}\right)$ si da cuenta del número de ocurrencias de una unidad léxica o relativa $\left(\mathrm{f}_{\mathrm{i}}\right)$ si se calcula sobre el tamaño de la muestra $\left(\mathrm{F}_{\mathrm{i}} / \mathrm{n}\right)$. En este trabajo consideraremos ambas frecuencias.

Otro índice relevante es el de dispersión compleja (D), que permite conocer cuán equilibrada es la distribución de las frecuencias en distintos subcorpus o mundos (Worlds, en Juilland y Chang-Rodríguez 1964: XXVI) que, en esta investigación, representan diferentes géneros discursivos. Para calcular la dispersión, hemos usado la fórmula de Juilland, Traversa, Beltramo, y Di Blasi (1973), que considera el número de mundos ( $\mathrm{n}=5)$, la suma de las frecuencias por mundo $\left(\mathrm{x}_{\mathrm{i}}\right)$ y la suma de las frecuencias totales (T):

$$
D=1-\frac{\sqrt{n x_{i}^{2}-T^{2}}}{2 T}
$$

Esta fórmula arroja resultados que van desde 0 (dispersión mínima) a 1 (dispersión máxima).

A partir de los índices de frecuencia y dispersión es posible obtener el de uso (U), consistente en el producto de la frecuencia -absoluta o relativa-por la dispersión. Puesto que los índices de dispersión y uso que empleamos en este trabajo han sido extraídos de Castillo Fadić (en referato), ambos se calculan a partir de frecuencias absolutas por dos razones: a) la primera, metodológica, radica en que se trabaja con subcorpus de tamaño homogéneo, lo que vuelve irrelevante estadísticamente la consideración del tamaño del mundo (n); b) la segunda, lexicográfica, estima que las frecuencias relativas, expresadas en decimales sin una parte entera, resultan menos amigables para los usuarios de un diccionario que las frecuencias absolutas, expresadas en números enteros. 
Los dos vocablos en estudio -mujer y hombre-, forman parte del Léxico Básico del Español de Chile, constituido por los alrededor de cinco mil vocablos de mayor uso en la comunidad, que se caracterizan por su distribución estable y por su tendencia atemática. Esta última característica es directamente proporcional al rango de uso o posición en que se ubican las unidades léxicas al ordenarlas de mayor a menor uso; este rango, a su vez, es inversamente proporcional a la dependencia de variables externas, como el género discursivo, el tema, el estilo, etc.

Tanto el vocablo mujer como el vocablo hombre presentan alta frecuencia $\left(\mathrm{F}_{\mathrm{i}}\right)$, dispersión (D) y uso (U) en el Corpus Básico del Español de Chile, por lo que ambos se ubican en los primeros rangos entre los sustantivos de mayor uso, como se aprecia en la Figura 1.

\begin{tabular}{|c|c|c|}
\hline Índices & Mujer & Hombre \\
\hline Frecuencia absoluta $\left(\mathrm{F}_{\mathrm{i}}\right)$ & 340 & 502 \\
\hline Dispersión compleja (D) & 0,73 & 0,8 \\
\hline Uso (U) & 248,2 & 401,6 \\
\hline Rango entre los sustantivos de mayor dispersión & 392 & 150 \\
\hline Rango entre los sustantivos de mayor uso & 12 & 7 \\
\hline
\end{tabular}

Figura 1. Índices estadísticos de mujer y hombre

\subsection{LEXICOSINTAXIS}

Si la estadística léxica permite establecer los índices estadísticos de las unidades léxicas, esta disciplina en interrelación con la lexicosintaxis, que observa las relaciones que se establecen entre un argumento y sus predicaciones, puede precisar también los índices combinatorios de dichas unidades. Esto es relevante, por cuanto las combinaciones frecuentes pueden revelar la presencia de locuciones u otras estructuras pluriverbales más o menos lexicalizadas. Al respecto, es importante precisar que incluso las estructuras de menor lexicalización, las llamadas colocaciones, que antaño se consideraron meras combinaciones frecuentes sin mayor intencionalidad y altamente transparentes, se entienden hoy mayoritariamente como "asociaciones entre un predicado y un conjunto restringido de argumentos que determinan una subclase léxica de mayor o menor extensión" (Bosque 2001: 17), puesto que la frecuencia de la combinatoria suele no ser arbitraria, sino estar sujeta a distintos factores lingüísticos y extralingüísticos. De hecho, "[f]uera de la fraseología existe un gran número de restricciones 
sintácticas y semánticas, incluso en las relaciones predicado-argumento". (Barrios Rodríguez 2016: 18)

\subsection{IDEOLOGÍA, CAMBIO LINGÜÍSTICO Y POLÍTICA LINGÜÍSTICA}

Concordamos con Ignacio Bosque (2012) -y con el pleno de la Real Academia Española que suscribe su informe- en que la lengua no es en sí sexista o no sexista, sino que el uso que se hace de ella es el que puede serlo o no. "El lenguaje puede usarse, en efecto, con múltiples propósitos. Puede emplearse para describir, ordenar, preguntar, ensalzar o insultar, entre otras muchas acciones, $\mathrm{y}$, desde luego, también puede usarse para discriminar a personas o a grupos sociales" (Bosque 2012). Coincidimos con él también en discrepar de quienes estiman que "el léxico, la morfología y la sintaxis de nuestra lengua han de hacer explícita sistemáticamente la relación entre género y sexo, de forma que serán automáticamente sexistas las manifestaciones verbales que no sigan tal directriz, ya que no garantizarían "la visibilidad de la mujer"". Nuestros reparos a esta postura se basan en la necesidad de no confundir sexo natural con género gramatical, en el principio de economía de las lenguas y, por último, en que intentar imponer una construcción morfológica de laboratorio a los hablantes, sea cual sea, implica, más allá de las buenas intenciones, caer en un prescriptivismo a ultranza, donde un grupo decide entre cuatro paredes cuál es la forma correcta de hablar, desestimando las otras y pretendiendo exiliarlas del ámbito público; algo similar, pero esta vez no en lo relativo al exilio de una variedad, sino al de una lengua, fue lo que intentó Franco con el catalán, y a la vista tenemos los resultados; no en vano ya Cervantes (2004: 872) ponía en boca de Don Quijote que "cuando algunos no entienden estos términos, importa poco, que el uso los irá introduciendo con el tiempo, que con facilidad se entiendan; y esto es enriquecer la lengua, sobre quien tiene poder el vulgo y el uso". En efecto: el vulgo y el uso; no individuos ni grupos particulares. De hecho, toda intervención lingüística que pretenda impactar en y desde las políticas públicas debe partir del conocimiento acerca de cómo evolucionan las lenguas y cómo se generan los procesos de cambio lingüístico en la historia (Cooper 1997). Como bien señala Alvar (2006),

[...] la lengua sigue siendo independiente por más que la sociedad pueda condicionarla; no se olvide que las motivaciones sociales actúan lentamente, sin llegar a romper lo que la lengua tiene de inmanencia. Cuando Stalin se enfrentaba con la realidad lingüística del ruso señalaba los cambios sociales que se habían producido en su país en algo más de 
cien años: desaparición del sistema feudal, liquidación del capitalismo, nacimiento del régimen socialista; se habían superado dos bases con sus supraestructuras y había surgido otra nueva con la suya propia, pero el ruso actual apenas si difiere en su estructura del ruso de Pushkin y poco después interroga: “¿Cómo se puede destruir la lengua existente y crear en su lugar, en unos pocos años, una lengua nueva sin llevar la anarquía a la vida social y sin provocar a la propia sociedad el riesgo de su disgregación?"

El cambio lingüístico, en efecto, no solo es resistente a las imposiciones, sino que además requiere de un tiempo considerable para asentarse. En esta línea, es altamente ilustrativo el caso del francés, mencionado por Moreno Fernández (2009: 118), en el que la pérdida de la /-n/ postnuclear y la nasalización de la vocal precedente tardó nada menos que cinco siglos.

Resumiendo, manifestamos tanto la ineficacia de intentar generar un cambio lingüístico rápido ( $c f$. Alvar 2006 y Moreno Fernández 2009), como la esterilidad de estos esfuerzos. Concordamos con Lemus (2001), en que el sexismo es un problema sociocultural, no lingüístico, por lo que su solución no pasa por intentar modificar la estructura de un idioma.

Proponemos, por último, la conveniencia de trasladar la atención del nivel morfológico al léxico; este nivel, el más cercano a la cosmovisión de los hablantes, es también el menos resistente al cambio lingüístico, por lo que puede ser el mejor comienzo no solo para estudiar la brecha de género, sino también para evaluar su potencial reducción. Es en el léxico, su uso y combinatoria, donde más claramente podemos observar tanto el pensamiento manifiesto como el que se esconde en el inconsciente colectivo.

\subsection{EL FACTOR SOCIAL}

En el caso de esta investigación, las combinaciones frecuentes de los sustantivos mujer y hombre podrían estar motivadas por factores extralingüísticos de corte social: específicamente, por la disímil valoración social de mujeres y hombres en Chile, que redunda en una mayor presencia masculina en la vida pública, no solo por sus mayores posibilidades de obtener y mantener un empleo bien valorado y remunerado, sino también por sus mayores ingresos y su mayor tiempo libre:

The gender gap in terms of jobs and earnings is large in Chile. Women earn on average around $20 \%$ less than men, are twice as likely to be in low-paid jobs, have a lower level of employment, and are more likely to be unemployed. However, Chilean girls fare slightly better than boys 
on tests of cognitive skills at 15 years of age. And while women feel less safe at night than men do, the gap between Chilean men and women is smaller than the OECD average gap. (OECD 2017: 4)

Adicionalmente, mientras los hombres en Chile dedican 34,6 horas semanales al trabajo remunerado y solo 19,0 al no remunerado, las mujeres solo cuentan con 19,8 horas de trabajo remunerado y destinan 42,1 a labores no remuneradas, especialmente en el hogar. En total, los hombres trabajan 53,6 horas a la semana, mientras las mujeres trabajan 61,9 horas, lo que supone no solo menos ingresos para ellas, sino también menos tiempo libre para el descanso, la vida social y la realización de actividades recreativas o de perfeccionamiento laboral, lo que disminuye su visibilidad en la vida pública. (CEPAL - Naciones Unidas 2016)

\section{METODOLOGÍA}

\subsection{OBTENCIÓN DEL CORPUS}

El Corpus Básico del Español de Chile es un corpus de referencia etiquetado de 501.211 palabras en contexto. Este corpus, que recibe este nombre porque fue la base a partir de la cual se obtuvo el Léxico Básico del Español de Chile (Castillo Fadić en referato), se distribuye en cinco mundos de alrededor de cien mil palabras cada uno, como se aprecia en la Figura 2. Ha sido obtenido de alrededor de cuatro mil textos de autores chilenos, publicados por primera vez en español como lengua original entre 1981 y 2006, distribuidos en casi mil libros y más de tres mil ejemplares de diarios de circulación nacional, que corresponden al menos al $15 \%$ del total de publicaciones por mundo o género discursivo en ese período. Para la obtención del corpus se realizaron diversos muestreos estratificados por mundo: a) primero, para precisar cuántas oraciones se requerían para obtener cien mil palabras por mundo; b) segundo, para determinar aleatoriamente de qué fuentes se extraerían las oraciones; c) tercero, para precisar las páginas; d) y cuarto, para seleccionar las líneas. En el caso de Prensa, los muestreos se estratificaron también por sección, para lo cual se consideraron las cuatro secciones comunes a todos los medios de circulación nacional del período, a saber, Opinión, Nacional, Deportes y Espectáculos. Para mayores informaciones sobre la obtención y constitución del corpus, $c f$. Castillo Fadić (2015: 3-5). 


\begin{tabular}{|c|c|}
\hline Mundos & Número de palabras \\
\hline Drama & 100220 \\
\hline Narrativa & 100323 \\
\hline Técnico-Científico & 100578 \\
\hline Ensayo & 100033 \\
\hline Prensa & 100057 \\
\hline Total & $\mathbf{5 0 1 2 1 1}$ \\
\hline
\end{tabular}

Figura 2. $\mathrm{N}^{\circ}$ de palabras por mundo

\subsection{Procesamiento del Corpus}

El corpus fue convertido a *.TXT, codificación ANSI, y se procesó con AntConc 3.2.4w.

Se realizó una búsqueda de las concordancias de mujer y hombre en los cinco mundos. Dado que las realizaciones de ambos vocablos contienen la base léxica mujer y hombre, respectivamente, para la búsqueda se seleccionó la opción Regex. Esto permitió obtener las concordancias de ambos vocablos (cf. Figuras 3 y 4 ).

\subsection{DESCRIPCIÓN Y ANÁLISIS DE LOS DATOS}

Los índices de frecuencia, dispersión y uso de ambos vocablos se obtuvieron de Castillo Fadić (en referato).

En AntConc 3.2.4w, se buscaron las combinaciones más frecuentes mediante un análisis por clusters en opción Regex, centrado en combinaciones de $F_{i} \geq 2$ de mínimo dos y máximo cinco unidades, situando a los dos vocablos en estudio a la izquierda del cluster, como se aprecia en las Figuras 5 y 6. Esto permitió generar un archivo output para cada vocablo, con las combinaciones léxicas más frecuentes de cada uno. A partir del análisis de las concordancias y de las combinaciones léxicas frecuentes obtenidas de la búsqueda por clusters, se abstrajeron las estructuras sintácticas en las que participa preferentemente cada vocablo en estudio, se evaluaron las posibles unidades fraseológicas o restricciones que les atañen y se determinó qué adjetivos, complementos o locuciones son los que más los determinan. 


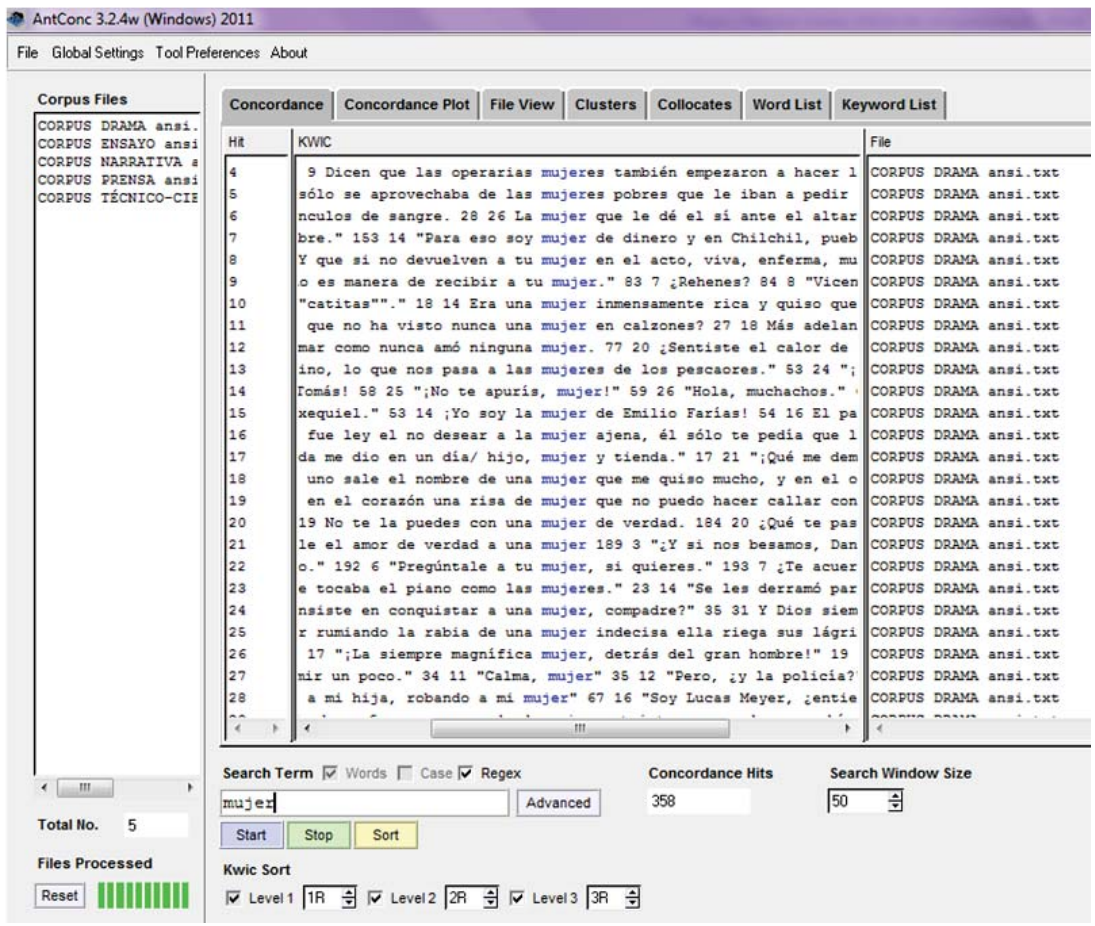

Figura 3. AntConc 3.2.4w, recorte de pantalla: concordancias mujer 


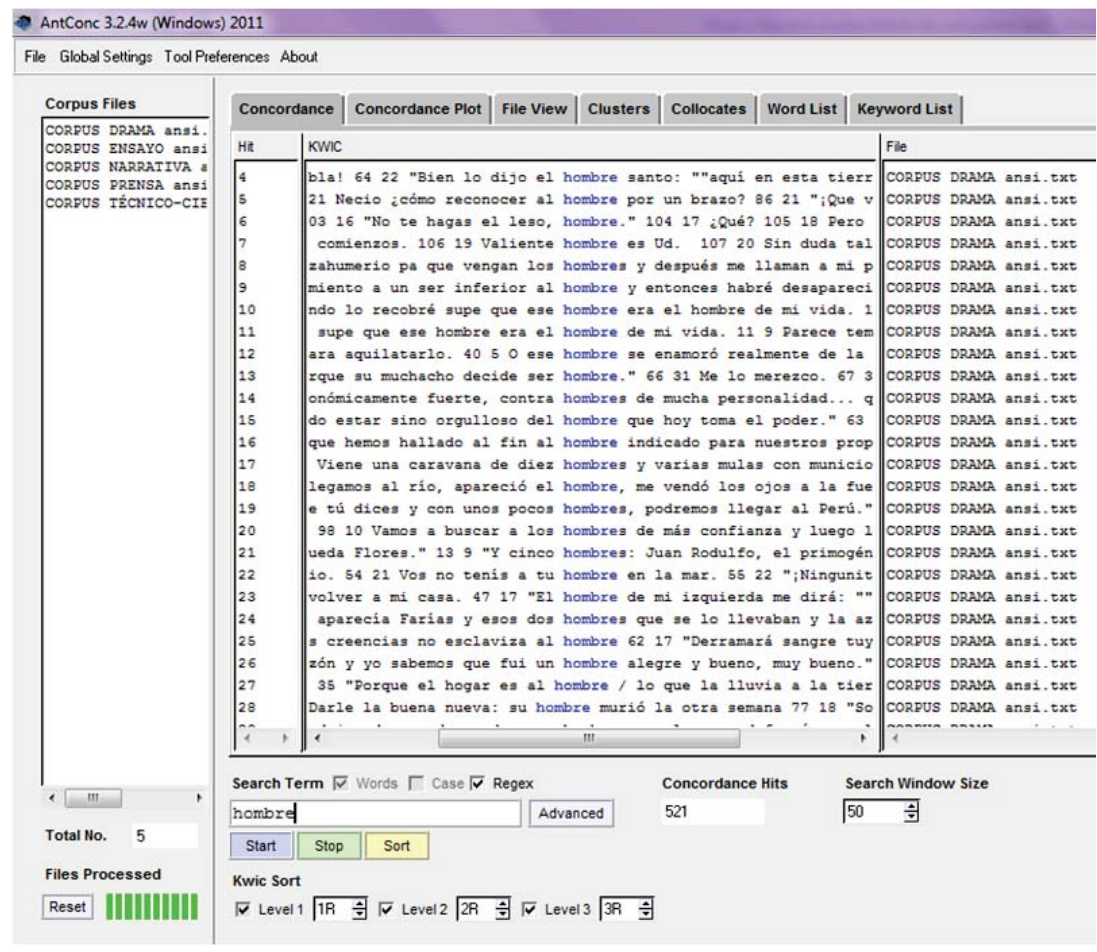

Figura 4. AntConc 3.2.4w, recorte de pantalla: concordancias hombre 
AntConc 3.2.4w (Windows) 2011

File Global Seltings Tool Preferences About

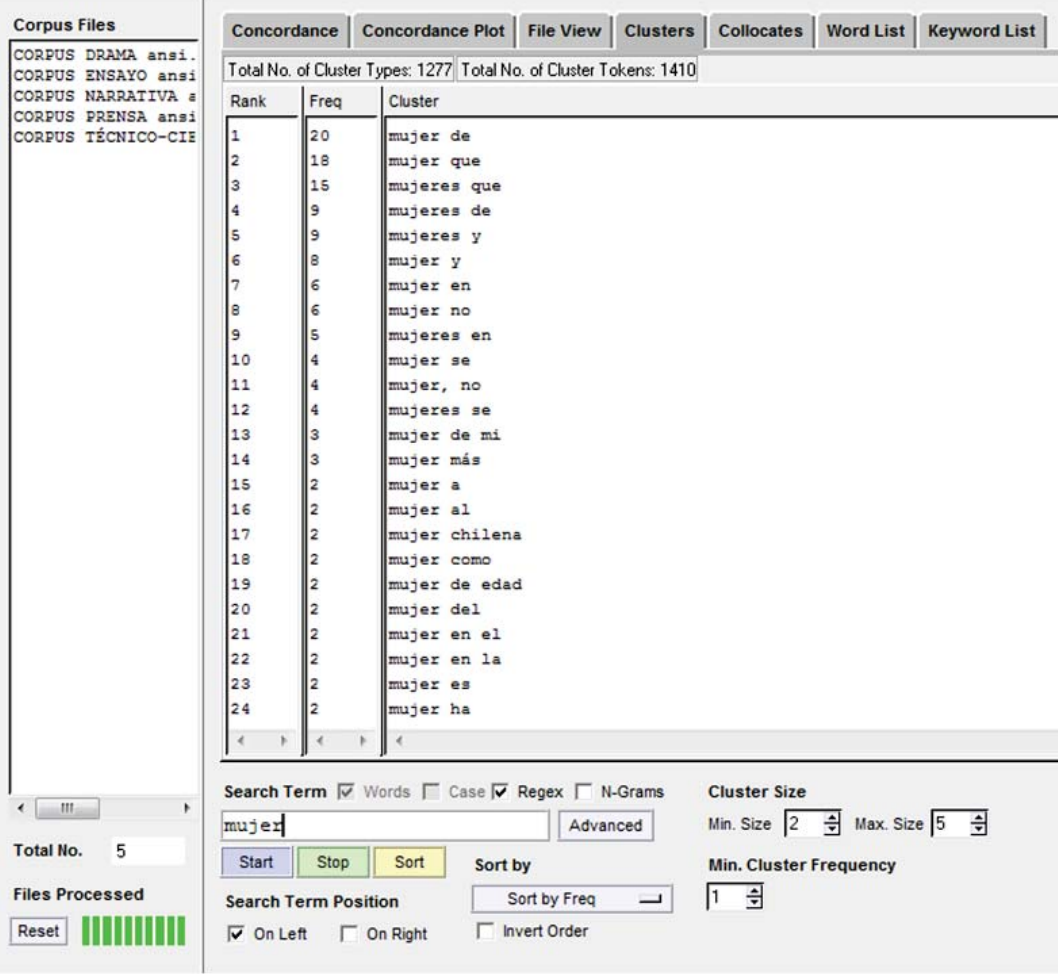

Figura 5. AntConc 3.2.4w, recorte de pantalla: análisis por clusters vocablo mujer 


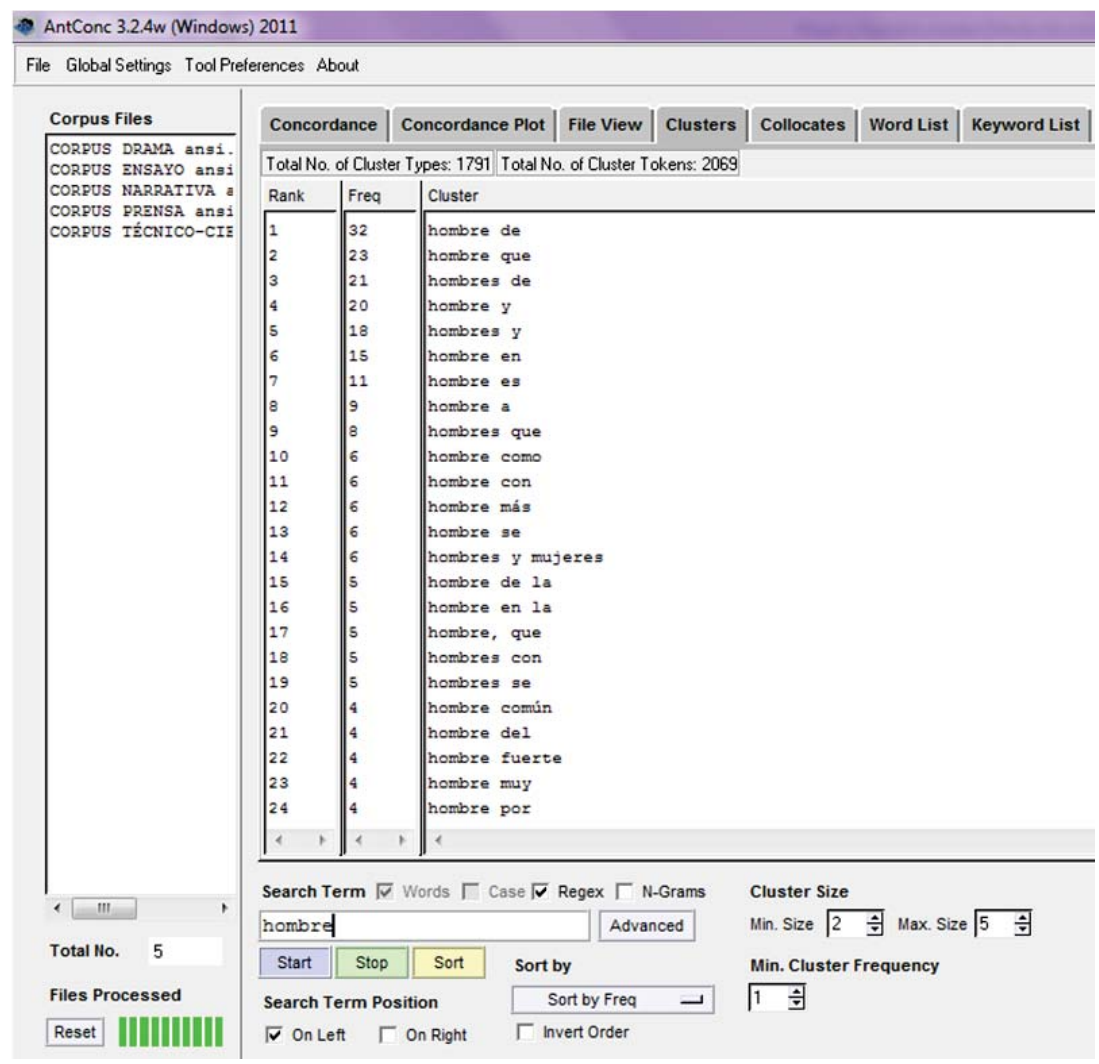

Figura 6. AntConc 3.2.4w, recorte de pantalla: análisis por clusters vocablo hombre

\section{RESULTADOS}

\section{1. ÍNDICES ESTADÍSTICOS}

Respecto de los índices de frecuencia, como se aprecia en las Figuras 7 y 8 , la $\mathrm{F}_{\mathrm{i}}$ de hombre es mayor a la de mujer en todos los mundos, lo que podría atribuirse, por un lado, a la mayor representación del ser humano masculino en los textos y, por otro, a la posibilidad del vocablo hombre de referirse al ser humano en general y no únicamente al de sexo masculino. Esta posibilidad podría explicar su mayor dispersión $-\mathrm{D}=0,8$ sobre $\mathrm{D}=0,73-$, ya que, como sabemos, la dispersión tiende a ser inversamente proporcional a la especificidad de un término. 
En la Figura 7 puede observarse que tanto la $\mathrm{F}_{\mathrm{i}}$ de hombre como la de mujer alcanzan sus mayores índices en Drama y los menores en Prensa. Sin embargo, mientras la proporción de ambos vocablos es similar en Drama, Narrativa, Técnico-Científico y Prensa, en Ensayo la $\mathrm{F}_{\mathrm{i}}$ de hombre prácticamente triplica a la de mujer, lo que queda claramente ilustrado en la Figura 8.

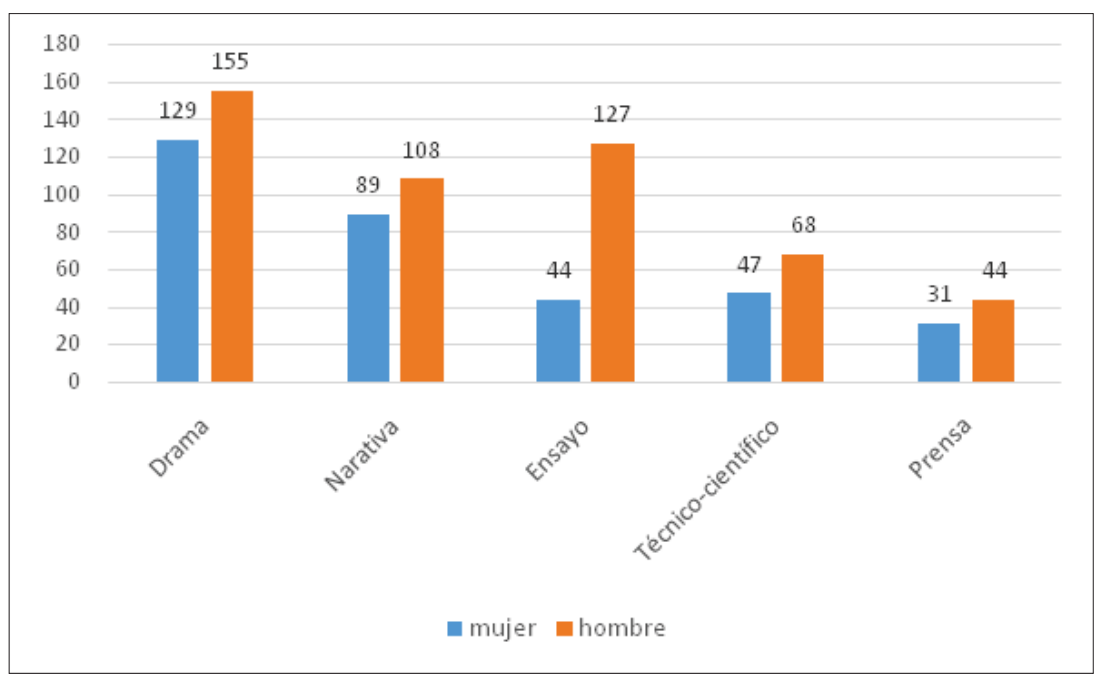

Figura 7. Distribución de vocablos mujer y hombre por mundo

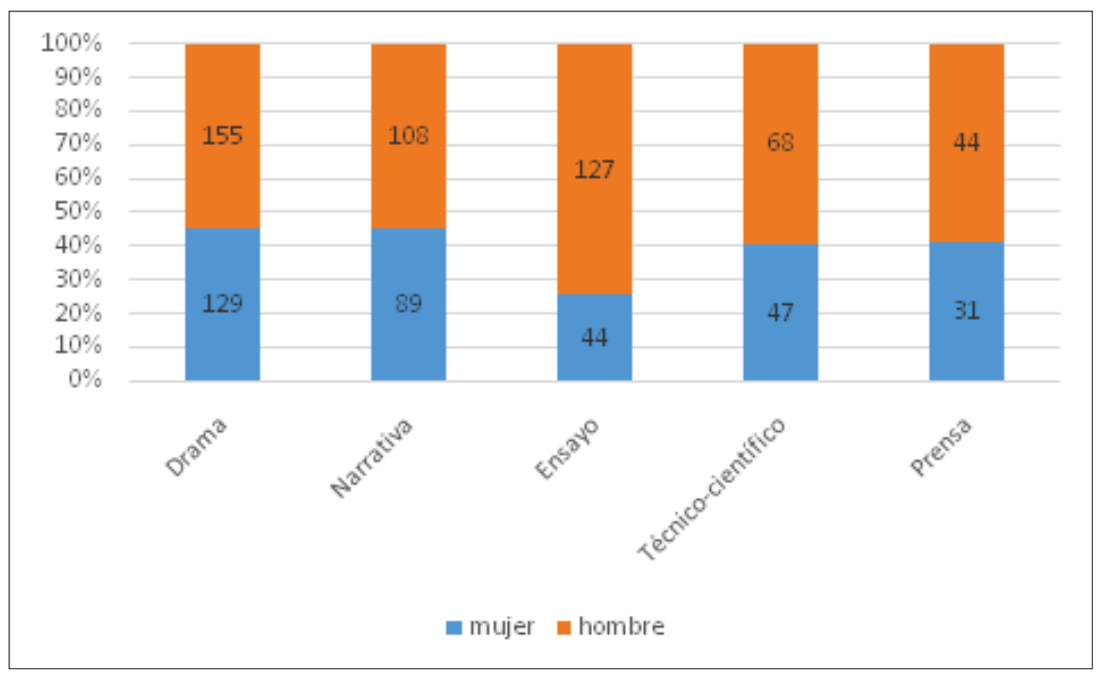

Figura 8. Proporción de vocablos mujer y hombre por mundo 
En efecto, si revisamos la distribución del vocablo mujer, un 38\% de las realizaciones aparece en Drama y un 26\% en Narrativa ( $c f$. Figura 9), lo que arroja un $64 \%$ de ocurrencias en textos de corte preferentemente literario. El porcentaje de realizaciones en Ensayo y en Técnico-Científico presenta diferencias estadísticamente marginales; ambos mundos sumados alcanzan un $27 \%$, es decir, menos de la mitad que Drama y Narrativa juntos. Prensa, por último, contiene solo el $9 \%$ de las realizaciones del vocablo.

En el caso del vocablo hombre, en cambio ( $c f$. Figura 10), un $31 \%$ de las realizaciones tiene lugar en Drama, un 25\% en Ensayo y un 21\% en Narrativa. Si consideramos que en Técnico-Científico se da un $14 \%$ de las realizaciones de hombre -mismo porcentaje que en ese mundo alcanza el vocablo mujer-, podemos observar que mientras el $52 \%$ de las ocurrencias de hombre se presenta en textos preferentemente literarios -Drama y Narrativa-, un elevado $39 \%$ tiene lugar en textos que tienden a ser especializados Ensayo y Técnico-Científico-, lo que contrasta con el $27 \%$ que en este tipo de textos alcanza el vocablo mujer. .

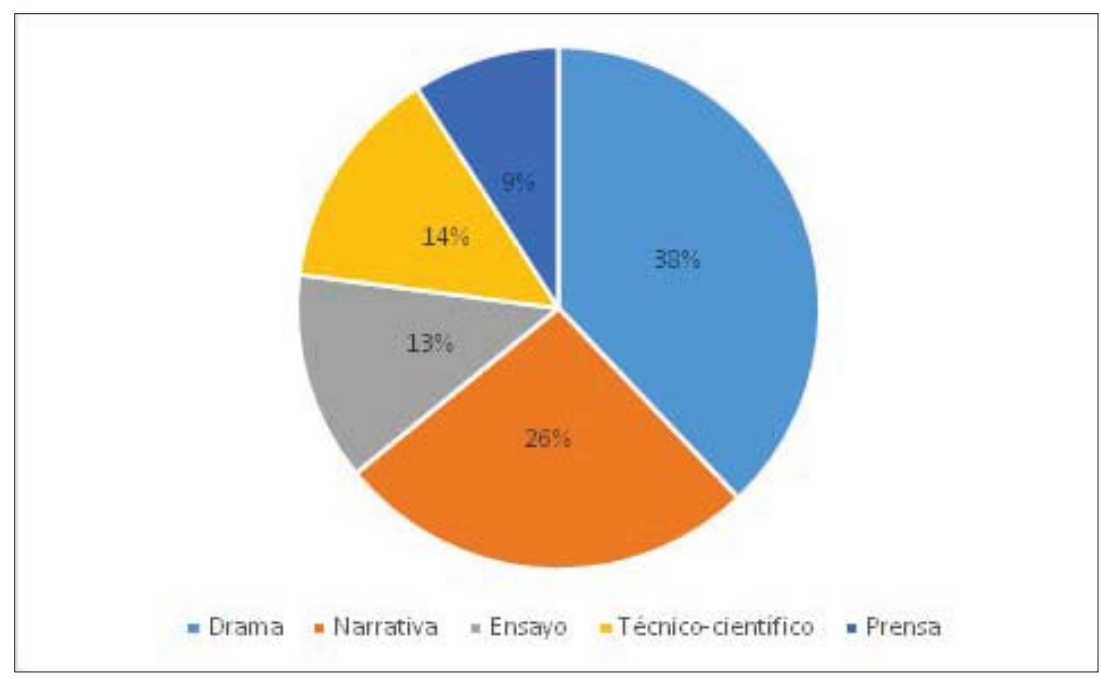

Figura 9. Distribución de mujer por mundo 


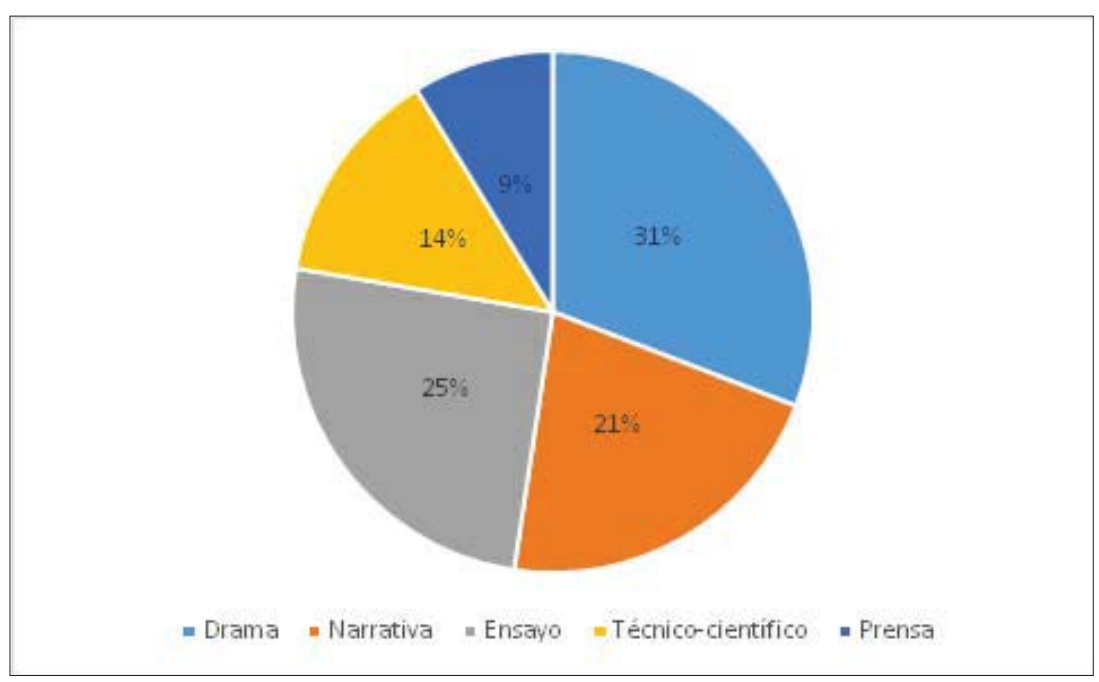

Figura 10. Distribución de hombre por mundo

\subsection{Combinaciones FRecuentes}

Ambos vocablos se combinan preferentemente con elementos subordinantes que introducen estructuras con función adjetiva ( $c f$. Figura 11).

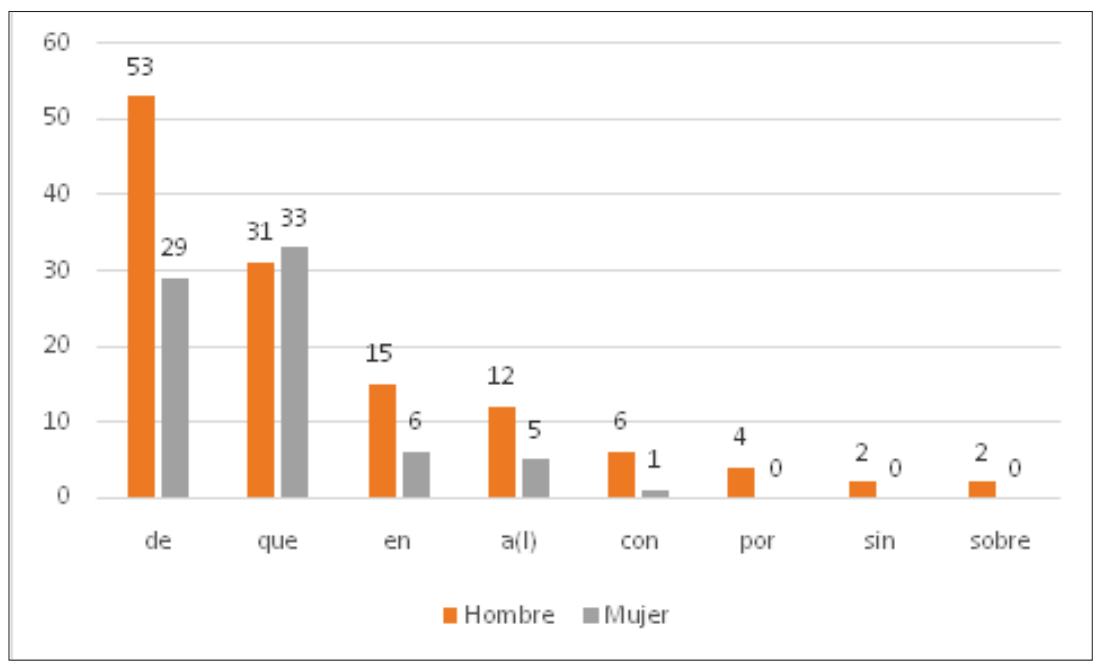

Figura 11. Elementos subordinantes que introducen estructuras con función adjetiva 
$\mathrm{Al}$ sumar las frecuencias por tipo de elemento subordinante ( $c f$. Figura 12), podría pensarse que mientras la combinación con prorrelativos -que introducen suboraciones adjetivas- es similar en ambos vocablos $-\mathrm{F}_{\mathrm{i}}=33$ en mujer y $\mathrm{F}_{\mathrm{i}}=31$ - en hombre, la combinación con preposiciones -que introducen complementos del nombre- es significativamente superior para hombre, con una $\mathrm{F}_{\mathrm{i}}=94$, más del doble de la $\mathrm{F}_{\mathrm{i}}=41$ de mujer.

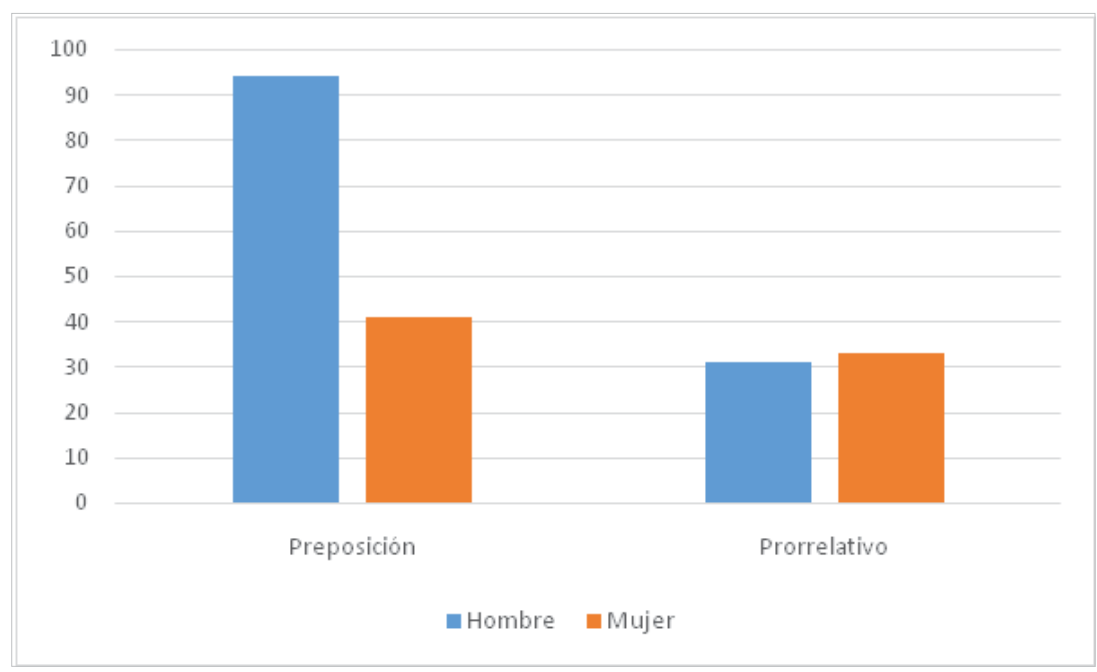

Figura 12. Combinación con prorrelativos y preposiciones (Fi)

Sin embargo, debemos recordar que la $\mathrm{F}_{\mathrm{i}}$ de ambos vocablos es disímil, por lo que para precisar las diferencias combinatorias es necesario trabajar con frecuencias relativas $\left(f_{i}\right)$, es decir, con aquellas que se calculan sobre el total de la muestra $\left(f_{i}=F_{i} / n\right)$. Al dividir la $F_{i}$ de la combinación de ambos vocablos con preposiciones y prorrelativos por el número de ocurrencias de mujer y hombre, podemos observar un cambio en los gráficos ( $c f$. Figura 13).

Si bien ambos vocablos continúan combinándose preferentemente con preposiciones $-\mathrm{y}$, por ende, con complementos del nombre-, la brecha que se apreciaba en la Figura 12 entre hombre + preposición y mujer + preposición decrece. Por otro lado, la combinación de mujer con prorrelativos -y, por tanto, con suboraciones adjetivas- casi duplica a la de hombre. 


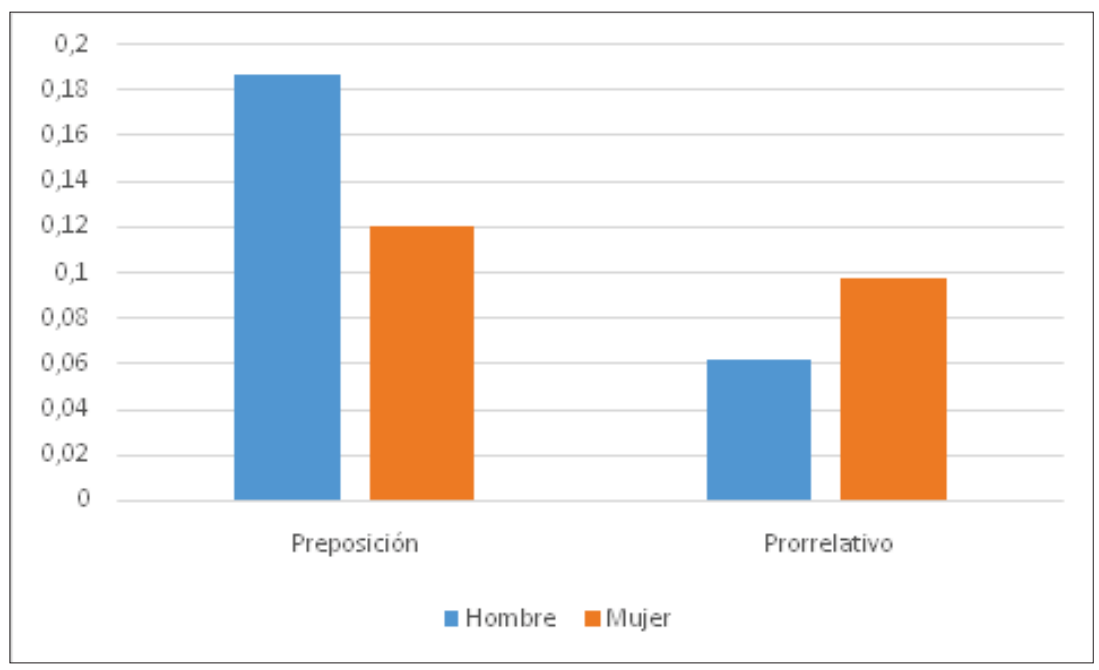

Figura 13. Combinación con prorrelativos y preposiciones (fi)

Pese a la elevada frecuencia combinatoria con elementos subordinantes que introducen complementos del nombre y suboraciones adjetivas, tanto los complementos como las suboraciones presentan alta variabilidad: mientras solo cuatro de los primeros alcanzan una $\mathrm{F}_{\mathrm{i}} \geq 2$, ninguna de las segundas logra una $\mathrm{F}_{i}>1$.

Los complementos que se reiteran predican en todos los casos de hombre y solo en un caso de ambos vocablos ( $c f$. Figura 14). A saber:

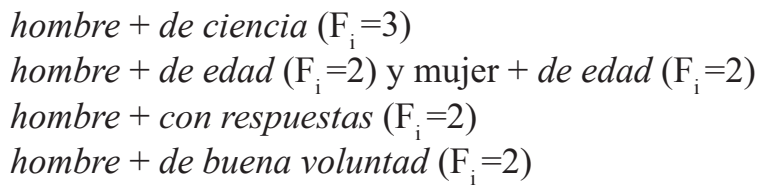

Los cuatro presentan cierto grado de lexicalización: de edad se encuentra incluso en RAE (2014) como locución adjetiva sin marca diatópica; de buena voluntad, otra locución adjetiva, está registrada en el mismo diccionario, pero solo como locución adverbial; de ciencia, no incorporada en la mencionada obra lexicográfica, es también a nuestro juicio una locución adjetiva; con respuestas parece tener un grado menor de fijación, por lo que podría clasificarse como colocación. 


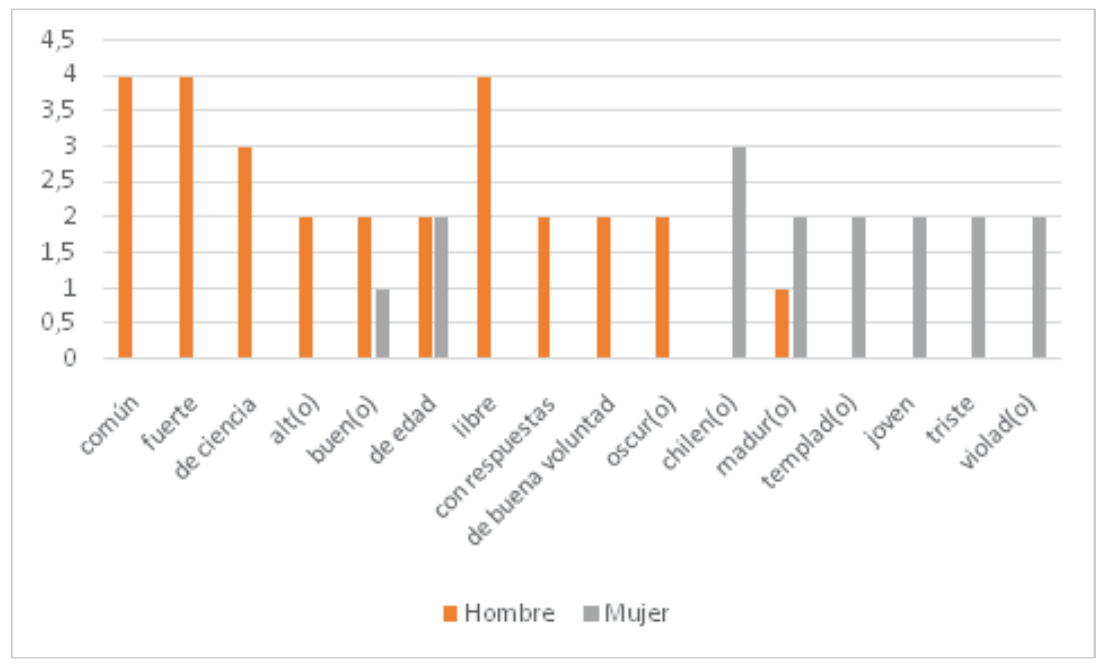

Figura 14. Modificadores del sustantivo con $\mathrm{F}_{\mathrm{i}} \geq 2$

$\mathrm{Al}$ revisar las locuciones adjetivas y la colocación identificadas ( $c f$. Figura 14), encontramos que las tres que predican exclusivamente de hombre-de ciencia, de buena voluntad y con respuestas - son positivas, mientras la que puede predicar de hombre o de mujer -de edad-es neutra.

Si observamos en la misma Figura 14 los adjetivos calificativos que predican de hombre y de mujer, podemos apreciar que solo dos de ellos se aplican a ambos vocablos, aunque con diferente $\mathrm{F}_{\mathrm{i}}$ : bueno (el doble para hombre) y maduro (el doble para mujer). En este último caso, el cotexto permite observar que maduro dicho de hombre da cuenta de una condición mental positiva, 'Que ha alcanzado la capacidad mental propia de una persona adulta' (RAE 2014, s. v. maduro, ra, acep. 3), mientras que maduro dicho de mujer se centra en una condición física negativa: 'Dicho de una persona: Que ha dejado de ser joven, pero no ha llegado a la vejez'.

Los diez adjetivos restantes -la mayoría- se aplican solo a hombre o solo a mujer (cf. Figura 15). Los que predican de hombre son preferentemente positivos -fuerte, alto, libre-. Los que predican de mujer no solo son preferentemente negativos -templada ('lujuriosa'), triste, violada-, sino que además incluyen dos unidades léxicas -templada y violada- que por su morfología de participio pasivo parecen dar cuenta de una mujer objeto, que recibe la acción realizada por otros. 


\begin{tabular}{|c|c|}
\hline Hombre & Mujer \\
\hline común & chilena \\
\hline fuerte & templada \\
\hline alto & joven \\
\hline libre & triste \\
\hline oscuro & violada \\
\hline
\end{tabular}

Figura 15. Modificadores del sustantivo con $\mathrm{F}_{\mathrm{i}} \geq 2$

\section{CONCLUSIONES}

\subsection{Alcances metodológicos}

El análisis automático de nuestro corpus de referencia nos ha permitido alcanzar los objetivos planteados y comprobar nuestras hipótesis. En una futura fase, consideramos conveniente complementar este trabajo con una mayor aplicación de métodos cualitativos.

\subsection{Principales Resultados obtenidos}

Se observa que los adjetivos, las locuciones y la colocación que modifican con más frecuencia a hombre y a mujer son diferentes y parecen responder a valoraciones más positivas del individuo humano masculino que del individuo humano femenino. Se confirman las tres hipótesis:

5.2.1. Alto número de modificadores nominales con $F_{i}=1$ y bajo número con $F_{i}>1$.

En efecto, por su alto uso, ambos vocablos presentan mínimas restricciones combinatorias, lo que se manifiesta en el escaso número de modificadores con $F_{i} \geq 2$. No obstante, se detectan dos estructuras que modifican frecuentemente a ambos sustantivos: complementos del nombre y suboraciones adjetivas de relativo. Además, se identifican tres locuciones adjetivas, una colocación y doce adjetivos que modifican a uno o a ambos vocablos con $F_{i} \geq 2$. 


\subsubsection{Más predicaciones con valor positivo para hombre}

Las tres unidades pluriverbales que predican solo de hombre son positivas. La que predica de hombre y de mujer es neutra.

Respecto de los adjetivos, cinco de los que predican de hombre son positivos: fuerte, alto, bueno, libre y maduro ('mentalmente adulto'). Sobre los que predican de mujer, solo dos son positivos: bueno y joven.

\subsubsection{Más predicaciones con valor negativo para mujer}

Solo hay un adjetivo con valor negativo que predica de hombre: oscuro ('sombrío'). En el caso de mujer, hay cuatro: templado ('lujurioso'), triste, violado y maduro ('que ha dejado de ser joven').

\subsection{TEMAS PARA INVESTIGACIONES FUtURAS}

A la vista de los resultados obtenidos de este estudio exploratorio, parece relevante analizar los adjetivos con $\mathrm{F}_{\mathrm{i}}=1$ que determinan a cada vocablo estudiado y determinar no solo su carga valorativa, sino también si dan cuenta de cualidades físicas, mentales, espirituales, etc. Esto permitiría precisar la preponderancia de una actitud positiva o negativa frente a los vocablos mujer y hombre como representaciones lingüísticas de construcciones culturales de género.

Resulta relevante, además, analizar las oraciones subordinadas de relativo que predican de mujer y de hombre, con énfasis en sus núcleos verbales. ¿Qué hacen los hombres y qué hacen las mujeres?

Estimamos de interés también determinar a qué se debe la mayor preponderancia relativa de hombre en el género Ensayo. ¿Obedece simplemente a un uso genérico (hombre $=$ individuo) o da cuenta de la menor presencia de la mujer como tema serio o de reflexión?; esto solo podría determinarse a partir de un análisis no automático de las unidades léxicas en contexto.

Por último, esta investigación podría extenderse a otros sustantivos con flexión de género cuyo género próximo sea 'individuo de la especie humana', como niño, niña, para indagar si los resultados obtenidos aquí, en cuanto a la mayor valoración del individuo masculino, se repiten. 


\section{REFERENCIAS BIBLIOGRÁFICAS}

Alvar, Manuel. 2006. Motivaciones sociológicas en el cambio lingüístico. Biblioteca Virtual Miguel de Cervantes [en línea]. Disponible en http://www.cervantesvirtual.com/obra-visor/ motivaciones-sociolgicas-en-el-cambio-lingstico-0/html/00eda5c4-82b2-11df-acc7002185ce6064_8.html\#I_0_[Consulta 12/09/2018].

Barrios Rodríguez, María Auxiliadora. 2016. Ignacio Bosque: gramático autor de diccionarios que nos hacen ser conscientes de todo lo que sabemos sobre las palabras. Paremia 25: 13-19.

Bosque, Ignacio. 2001. Bases para un diccionario de restricciones léxicas. Moenia 7: 11-52. 2012. Sexismo lingüístico y visibilidad de la mujer. Real Academia Española [en línea]. Disponible en: http://www.rae.es/sites/default/files/Bosque_sexismo_linguistico. pdf [Consulta 27/07/2018].

Castillo Fadić, María Natalia. 2012. Corpus Básico del Español de Chile (C).

2015. Léxico Básico del Español de Chile: el proyecto. E-Aesla. Revista digital [en línea]. Disponible en: https://cvc.cervantes.es/lengua/eaesla/pdf/01/51.pdf [Consulta 24/04/2017].

(en referato.). Léxico Básico del Español de Chile.

CEPAL - Naciones Unidas. 2016. Tiempo total de trabajo. Observatorio de Igualdad de Género de América Latina y el Caribe [en línea]. Disponible en: https://oig.cepal.org/es/ indicadores/tiempo-total-trabajo. [Consulta 15/08/2018].

Cervantes, Miguel De. 2004. Don Quijote de la Mancha. San Pablo (Brasil): Real Academia Española y Asociación de Academias de la Lengua Española.

COOPER, RoBert. 1997. La planificación lingüística y el cambio social. Madrid: Cambridge University Press.

Juilland, Alphonse y Eugenio Chang-Rodríguez. 1964. Frequency Dictionary of Spanish Words, The Romance Languages and their Structures, First Series SI. La Haya: Mouton.

Juilland, Alphonse, Vicenzo Traversa, Antonio Beltramo y Sebastiano Di Blasi. 1973. Frequency Dictionary of Italian Words. The Hague- Paris: Mouton.

Lemus, Jorge Ernesto. 2001. Sexismo en el lenguaje: mitos y realidades. Memorias del Encuentro de la Red Centroamericana de Antropología, Asociación Salvadoreña de Antropología, pp. 195-225.

Manrique Sabogal, Winston. 5 de marzo de 2012. ¿La lengua tiene género? ¿Y sexo? El País [en línea]. Disponible en: https://elpais.com/cultura/2012/03/04/ actualidad/1330896843_065369.html [Consulta 08/12/2017].

Marín, Pablo. 24 de junio de 2018. ¿Todes juntes? El lenguaje inclusivo como batalla cultural. La Tercera. Disponible en: https://www.latercera.com/tendencias/noticia/ todes-juntes-lenguaje-inclusivo-batalla-cultural $/ 218599 /$ fbclid=IwAR2GsS0 $0 Y$ ao_CERAvzeSsdhTmgh3TMzTvUWM3kwu-wG48L6hJChSNOXK40 [Consulta $27 / 06 / 2018]$.

Moreno Fernández, Francisco. 2009. Principios de sociolingüística y sociología del lenguaje ( $4^{\mathrm{a}}$ ed). Madrid: Ariel.

OECD. Noviembre de 2017. How's Life in Chile? OECD.org [en línea]. Disponible en: https://www.oecd.org/statistics/Better-Life-Initiative-country-note-Chile.pdf [Consulta 10/05/2018].

Real Academia Española (RAE). 2014. Diccionario de la Lengua Española [en línea]. Disponible en: http://www.rae.es/diccionario-de-la-lengua-espanola/la-23a-edicion-2014 [Consulta 01/05/2018]. 